\title{
Unterricht zwischen Schule und Zuhause: Perspektiven von Schulanfänger*innen auf Lernprozesse im Homeschooling
}

\author{
Birgit Hüpping, Mirja Kekeritz und Melanie Kubandt
}

\section{Zusammenfassung des Beitrags}

Die vorliegende qualitative Studie untersucht Perspektiven von Schulanfänger*innen aus einer gesamten Grundschulklasse im Wechsel von Homeschooling- und Präsenzphasen. Die Studie ist gekennzeichnet durch zwei Erhebungszeiträume in den durch die Pandemie geprägten Zeiträumen 2020 und 2021. Im Zentrum des vorliegenden Beitrags steht die erste Lockdownphase (Frühjahr/Sommer 2020). Die rekonstruierten Herausforderungen und Bewältigungsstrategien der Kinder beim schulischen Lernen im familialen Setting werden dahingehend beleuchtet, welche Rolle mediales Lernen spielt und welche Rolle der Einsatz digitaler Medien hier perspektivisch spielen könnte.

Schlüsselbegriffe: Corona $\bullet$ Homeschooling $\bullet$ Schulanfang $\bullet$ Grundschule $\bullet$ Kindheitsforschung

\section{Einleitung}

Die Covid-19-Pandemie hat im Zusammenhang mit dem kurzfristig implementierten Distanzunterricht zwischen Schule und Zuhause sowohl Lehrer*innen als auch Schüler*innen unerwartet herausgefordert und die Frage nach der Relevanz digitaler Angebote im schulischen Kontext noch deutlicher als zuvor in den Fokus gerückt. Die unvorhersehbare Umstellung auf Homeschoolingphasen ${ }^{1}$ machte kurzfristige Lösungen und Anpassungen im Hinblick auf die Unterrichtsplanungen und -gestaltungen seitens Lehrpersonen zwingend notwendig. Es kann vermutet werden, dass auf Seite der Schüler*innen insbesondere Schulanfänger*innen (Einschulung Sommer 2019) hier spezifisch herausgefordert waren, da sie diese veränderten, neuen Unterrichtsformen im heimischen Umfeld zu einem Zeitpunkt getroffen haben, an dem schulische und unterrichtliche Logiken noch relativ neu für sie waren und die Transition in die Grundschule im Sinne einer „Schwellen- und Umwandlungsphase“ (vgl. van Gennep 2005, S. 238) noch nicht abgeschlossen war. Vor dem Hintergrund der pandemiebedingten

\footnotetext{
${ }^{1}$ Der (digitale) „Fernunterricht“ (Fickermann/Edelstein 2020a) oder Distanzunterricht wird alltagssprachlich - insbesondere im medialen Diskurs rund um die Themen Pandemie, Bildung und Familie (vgl. u.a. Anders 2021) - häufig auf den Begriff „Homeschooling“ heruntergebrochen. Im Rahmen dieses Beitrags findet mitunter auch der alltagssprachliche Begriff „Homeschooling“ Verwendung, um Begriffe des Feldes aufzugreifen und zuletzt auch auf die hier sehr bedeutsame häuslich-familiäre Einbettung schulischen Lernens zu verweisen.
} 
Anforderungen an eine Umstellung der Unterrichtsgestaltung in Formate, die von den Kindern im familiären Umfeld sinnvoll und pädagogisch angemessen realisiert werden können, widmet sich dieser Beitrag daher den Erfahrungen und Perspektiven von Schulanfänger*innen im Homeschooling der ersten Lockdownphase (Frühjahr/Sommer 2020). Das hier vorgestellte Projekt umfasste zwei Erhebungszeiträume (2020 und 2021) und fokussiert in diesem Beitrag die Erfahrungswerte der Kinder aus der ersten Erhebung zu Beginn der Umstellungen zwischen schulischem und häuslichem Unterricht. Daran lässt sich rekonstruieren, wie herausfordernd es ist, schulische Logiken in das häusliche Umfeld zu übertragen und inwiefern digitale Medien hierbei eine Rolle spielen. Zu diesem Zweck wird zunächst in Abschnitt 1 in den spezifischen Kontext und die sozialkonstruktivistische Verortung der qualitativen Interviewstudie in einer zweiten Schulklasse eingeführt. In Abschnitt 2 steht anschließend das konkrete Forschungsdesign im Fokus der Ausführungen, das in die Forschungserkenntnisse in Abschnitt 3 überleitet. Dabei fokussieren wir spezifisch auf Herausforderungen und Bewältigungsstrategien von Zweitklässer*innen während bzw. nach der ersten Lockdownphase im Hinblick auf schulisches Lernen im familialen Setting. Der Beitrag schließt vor dem Hintergrund der rekonstruierten Erkenntnisse u.a. mit Überlegungen zu einem integrativen Einsatz digitaler Medien und damit verbundenen Lernangeboten in Homeschoolingphasen an.

\section{Hintergrund der Studie}

Das dynamische Forschungsfeld Corona und Schule setzt sich mit seiner jungen Forschungsgeschichte mit den pandemiebedingten Umstellungen und Einschränkungen von Unterricht und Schulalltag sowie mit dem Fernunterricht und Präsenzunterricht mit seinen verschiedenen Varianten (Hybridunterricht) auseinander (vgl. Fickermann/Edelstein 2020a). In diesem Zusammenhang werden in entsprechenden Debatten auch wiederholt Fragen der Digitalisierung sowie der Einsatz digitaler Angebote im Homeschoolsetting zum Thema. Nicht erst seit den Herausforderungen in den Pandemiejahren 2020/2021 besteht auch für die Grundschule das Ziel, Medienkompetenzen sowohl aufSeiten der Lehrkräfte als auch der Schüler*innen u.a. im Hinblick auf den Umgang und den Einsatz von digitalen Medien zu etablieren. Bereits die Hälfte der 6- bis 13-Jährigen besitzen Mobiltelefone und verfügen über die Fähigkeiten, „Nachrichten, Fotos oder Videos [zu] versenden“ oder „im Internet zu surfen“ (MPFS 2018, S. 16, vgl. auch MPFS 2020). Doch je nach soziokulturellen Merkmalen der Kinder und Jugendlichen sind Unterschiede darin zu erkennen, inwieweit medial und digital vermittelte Informationen verstanden, genutzt und kommuniziert werden (vgl. u.a. Eickelmann 2019). Daher ist es u.a. laut eines Strategiepapiers der Kultusministerkonferenz (vgl. KMK 2017) notwendig, dass bereits im Grundschulalter Grundlagen zum Kompetenzerwerb für eine aktive, selbstbestimmte Teilhabe in einer digitalen Welt geschaffen werden. Langfristige Perspektive ist es, Lehr- und Lernprozesse unter den Bedingungen der digitalisierten Welt grundlegend zu verändern. Als zwei zentrale Zielsetzungen auf diesem Weg werden zum einen die curriculare Verankerung von digitaler Medienkompetenz, zum anderen die Verstärkung bzw. der Ausbau von Lehr-Lern-Prozessen unter den Bedingungen der Kultur der Digitalität seitens der KMK gesetzt. Hierbei ist laut Schaumburg (vgl. 2019) bei der didaktischen Planung und der konkre- 
ten Realisierung von Unterrichtseinheiten zwischen dem spezifischen Mehrwert sowie technischen und didaktischen Herausforderungen abzuwägen. Irion und Scheiter (vgl. 2018) verweisen des Weiteren auf drei Aspekte, die das Potenzial des digitalen Medieneinsatzes im Grundschulunterricht stets hinsichtlich seiner pädagogischen Funktion hinterfragen sollten: Präsentationsformen (Dienen die gewäblten Tools der Veranschaulichung von Unterrichtseinheiten?), Individualisierung (Werden beispielsweise adaptive Unterrichtsformen unterstützt?) und Kooperation (Dient der Einsatz der digitalen Medien kooperativen Unterrichtsformen?).

Während die Digitalisierung bereits seit längerem schulische Debatten prägt, wirken die aktuellen Entwicklungen in Lockdownphasen mit kurzfristigen Umstellungen auf Homeschooling wie ein Beschleuniger, der unterschiedlichste Herausforderungen einer Umstellung auf digital gestützte Lernprozesse offenlegt. Die vielfach zitierte Metapher des Brennglases, stellvertretend für dynamische Veränderung in Zeiten von Corona, lässt sich auch für die Transformation der Institution Grundschule heranziehen, um die dortige Lernkultur in ihrer Variation zu erfassen und zugleich verallgemeinerbare, übergreifende Veränderungen des Schulischen zu erkennen (vgl. Idel et al. 2013, S. 249). Die angewandten Lernformate in Phasen von Distanz- und Wechselunterricht führen nicht zu einer identischen Reproduktion bisheriger schulischer Lernpraktiken, da hier Übersetzungsleistungen von dem Bildungs- und Lernverständnis der Kinder und ihrer Familien abhängig sind. Hierbei ist generell auch das schultypische Zusammenspiel von organisationalen und pädagogischen Diskursen und Praktiken zu betrachten (vgl. Idel et al. 2013, S. 249), die Einfluss auf Schulentwicklungsprozesse nehmen, wie z.B. die Einbindung der Schlüsselkompetenz Medienbildung und damit verbundener digitaler Prozesse auf der Schul- und Unterrichtsebene.

Vor dem Hintergrund der aktuellen Covid-19-Pandemie fokussiert die vorliegende Studie daher, wie die Rückkehr in die Schule nach der ersten Homeschoolingphase (und langen Ferienzeiten) als auch der Wechsel zwischen Präsenzunterricht und Homeschooling von Zweitklässler*innen (Einschulung Sommer 2019) wahrgenommen wird. Eine Besonderheit der vorliegenden Studie ist, dass eine komplette Schulklasse untersucht wurde und spezifisch die Schüler*innenperspektiven im Zentrum stehen. Des Weiteren ist die Fokussierung auf eine Grundschulklasse auch deswegen aufschlussreich, da sich insbesondere in Grundschulen große Herausforderungen, die mit der Distanzierung von Unterricht und der Digitalisierung des Lernens einhergehen, zeigen (vgl. Huber et al. 2020, S. 63f.; Ravens-Sieberer et al. 2021). Der Einsatz digitaler Lehr-Lernformen ist für Grundschüler*innen bezüglich der „Entwicklung sozio-emotionaler Kompetenzen und des Austauschs untereinander [...] eingeschränkter möglich“ (Huber et al. 2020, S. 109). Hier hat sich beispielsweise im Schulbarometer (ebd.) gezeigt, dass „(j)e tiefer die Schulstufe, desto größer (ist) der Anteil analogen Arbeitens“ (Huber et al. 2020, S. 28). Demnach liefert der differenzierte Blick darauf, wie sich der Wechsel von Homeschooling- und Präsenzphasen von Unterricht einer zweiten Klasse aus Sicht der Schüler*innen gestaltet, auch dahingehend Aufschluss, die Bedarfslage von Schüler*innen, deren Erleben von Chancen und Belastungen und damit verbundene Unterstützungsbedarfe zu erkennen: Welche schulischen Alltagsstrukturen haben sich aus Perspektive der Zweitkläss- 
ler*innen neu etabliert oder verändert? Welche aktiven Bewältigungs- und Gestaltungsstrategien lassen sich auf Seiten der Kinder (im Homeschooling und in der Phase der Schulrückkehr) rekonstruieren?

Die Zweitklässler*innen sind dabei im sozial-konstruktivistischen Verständnis als soziale selbstständige Akteur*innen zu verstehen, die den institutionellen Rahmen ko-konstruieren (vgl. de Boer/Deckert-Peaceman 2009b). Der „fluide Charakter des Peerseins und die Rolle des Lernenden/der Lernenden und seine Kontextgebundenheit“ (Kekeritz 2017, S. 221) werden durch die besonderen medial-räumlichen Veränderungen der Pandemie in neue Verhältnisse gebracht, die es zu beleuchten gilt. In Rückgriff auf ein relationales Verständnis von Agency (vgl. Eßer 2014) lässt sich Schule dabei als ein dichtes Netzwerk von Beziehungen darstellen, das durch vielfältige Interaktionen schulischer Akteur*innen - Erwachsene wie Kinder - geprägt ist (vgl. Hüpping/Kamin 2020, S. 130). Die Interaktionen beziehen sich dabei auch auf sogenannte nicht-menschliche Akteur*innen als Aktanten bzw. Dinge (vgl. Eßer 2014; Nohl 2011) in Form von analogen und digitalen Medien schulischen Lernens. Diese Relationalität ermöglicht es, schulische Praktiken als fortlaufende Reproduktions- und Produktionsprozesse sozialer Ordnungen zu identifizieren, die eng verwoben sind mit mentalen Konstruktionen der an Schule beteiligten Akteur*innen. Heinzel (vgl. 2019) hebt daher die soziale Praxis der Grundschule als Beziehungen zwischen den Generationen hervor und betont die Handlungsfähigkeit und -mächtigkeit der Kinder. Erst durch die Teilhabe werden die Kinder mit einem spezifischen Verständnis von sich selbst als Akteur*innen - und damit auch als Subjekte - ausgestattet und betreiben situativ hochvariabel Selbstpositionierungen (vgl. Bollig/Kelle 2014, S. 274). Die Studie fasst demnach die Praktiken der Kinder im Kontext von Peerkulturen und institutionellen Rahmungen (wie Unterricht) als Teil der institutionellen Ordnungen auf und hebt damit die „sozio-kulturelle Dimension des Lernens und damit ihre Bedingtheit von sozialen Ordnungsprozessen" (de Boer/Deckert-Peaceman 2009a, S. 14) hervor.

Vor diesem Hintergrund stellt sich die Frage: Wie werden Praktiken schulischen Lernens im familiären Umfeld aufgegriffen, übersetzt und angewandt und wie verschiebt sich - durch den institutionellen, schulischen Zugriff - ihre Bedeutung in Phasen von mediengestütztem Distanzlernen in der Familie? Damit verbunden ist zum einen die Frage, ob die Schüler*innen Möglichkeiten des selbstbestimmten Lernens mit kreativen Möglichkeiten, eigenem Lernrhythmus und -tempo wie auch individuellen Lernweisen nutzen, zum anderen, inwiefern sie die pandemiebedingte Situation als belastend erleben und welche Unterstützung sie von Lehrkräften und anderen Akteur*innen erhalten wie auch benötigen. Dabei fokussierte die erste Erhebungsphase der Studie die Anfangsphase der Pandemie bzw. des Lernens zwischen Homeschooling- und Präsenzphasen und nimmt die anfänglichen Digitalisierungs- und Umstellungsprozesse in den Blick.

\section{Methodisches Vorgehen}

Die qualitative Studie wurde mit der gesamten Schülerschaft $(n=21)$ einer zweiten Grundschulklasse im Raum Ludwigsburg (Baden-Württemberg) im November 2020 durchgeführt. Das Forschungsdesign umfasst zwei Komponenten, um im Sinne des Verständnisses von 
Kindern als aktive Konstrukteure ihrer Selbst, ihrer Lebenswelt und Kindheit verschiedene Zugänge zu kindlichen Perspektiven methodologisch wie methodisch zu berücksichtigen: Im Rahmen des Sachunterrichts wurden die Kinder über das Forschungsprojekt von einer Forscherin aufgeklärt und eingeladen, einen Fragebogen auszufüllen, forschungsinduzierte Kinderzeichnungen zu erstellen oder über ein ausgelegtes Diktiergerät Audiostatements in Form eines non-reaktiven Verfahrens (vgl. Hartnack 2019) zu geben. Im Kern des Fragebogens standen hierbei das persönliche Erleben des Homeschoolingalltags und das Wohlbefinden bei der Rückkehr in den von AHA-Regeln geprägten Schulalltag. Es wurden geschlossene Fragen mit text- sowie symbolbasierten (Smileys) Antwortvorgaben und wenige offene Frageformate genutzt. Die Kinderzeichnungen wurden durch Schreib-Mal-Blätter mit der Überschrift „Ich beim Lernen im Homeschooling“ oder "Ich wieder in der Schule“ oder „Corona und Schule: So komme ich damit klar..." induziert und bezogen sich auf die lebensweltlichen Erfahrungen der Kinder. Sie werden im Rahmen der Studie als produktive Selbstäußerungen der Kinder mit ästhetischem Eigensinn aufgefasst, wobei der von den Forscher*innen als auch der Schule gerahmte Entstehungs- und Produktionskontext der Kinderzeichnungen zu beachten ist (vgl. Konecki 2011).

Dieses Angebot stand über einen Zeitraum von zwei Wochen zur Verfügung und Kinderzeichnungen wie Fragebögen konnten anonym in eine Postbox eingeworfen werden. Die Inhalte der Postbox wurden anschließend von der Feldforscherin gemeinsam mit den Kindern im Zuge der Sachunterrichtseinheit gesichtet und deskriptiv ausgewertet. An dieser Stelle ist kurz anzumerken, dass die Datenerhebung via Postbox bzw. Auswertung dieser Daten mit den Kindern eine doppelte Zielsetzung verfolgte: Einen partizipativen Charakter, um gemeinsam mit den Kindern ibre Daten zu sichten und einzuordnen. Zudem fungierte die Auswertung auch als kommunikative Validierung der gewonnen Daten für die Forscherinnen. Die Auswertung erfolgte auf der organisatorischen Ebene gemeinsam mit der Klassenlehrerin in Kleingruppen, wobei der Prozess und die damit verbundene Kommunikation der Kinder in Auseinandersetzung mit den Daten im Vordergrund stand. Abschließend stellten die Kinder ihre Eindrücke und Ergebnisse der Klasse vor. Diese Verknüpfung von Forschungsgegenstand und forschendem Lernen in der Schule (vgl. Knörzer et al. 2019) erfüllen eine didaktische sowie methodologische Funktion, da sie einerseits auf den individuellen und kollektiven Erkenntnisgewinn der Kinder zielen und zum anderen im partizipativen Sinne in den Diskurs mit den Kindern während Datenerhebung wie auch -auswertung treten.

Zeitnah zur ersten Erhebung wurde (vor der gemeinsamen Auswertung) die gesamte Klasse in Partnerkonstellationen zu Interviews eingeladen. Das offene Leitfadeninterview deckte konkrete Erfahrungen und Routinen der Kinder zum Homeschooling, zum Übergang zurück in die Schule und den neuen Schulalltag unter Pandemiebedingungen ab. Diese - also einem Mixed-Methods-Design entstammenden - Daten wurden in einem zirkulären Forschungsprozess in Orientierung an der Grounded Theory (vgl. Glaser/Strauss 1998) segmentanalytisch ausgewertet. Da weitere Erhebungen erfolgen, ist die zirkuläre Verquickung von Datengewinnung, -analyse und theoretischen Arbeiten weiterhin im Prozess. 


\section{Erkenntnisse der Studie}

Stellenwert analoger und digitaler Medien

Der Einsatz analoger und digitaler Medien ist eng verwoben mit inhaltlichen und organisatorischen Rahmenbedingungen. Mit Blick auf die Organisation benennen die Kinder, dass sie zu Beginn der Schulschließungen ihre Aufgaben persönlich erhalten haben. Ergänzende Informationen zum Wochenplan wurden direkt an die Mailkontakte ihrer Eltern adressiert. Mit zunehmender Dauer des Homeschoolings verweisen die Kinder auf den Einsatz des Lernmanagementsystems Moodle. Inhaltlich überwiegt die Nutzung analoger Medien, während digitale Formate wie der Einsatz von Videos, Apps und Lernspielen sich auf Mathematik, Sport und Musik fokussieren.

B3: [...] ich habe immer nach dem Stundenplan gelernt, den mir die Frau (Name der Klassenlehrerin) mitgeschickt hat mit den Hausaufgaben oder Aufgaben besser gesagt. Ähm habe ich auch, war ich auch immer klargekommen und (..) ich habe dann immer auch dann bisschen im Rechenfuchs Indianerheft gearbeitet und dann habe ich in der Anton-App weitergemacht.

Die Erkenntnisse aus der ersten Erhebungsphase der vorliegenden Studie zeigen die bisherigen, prä-pandemisch geprägten Alltagspraktiken im Schulkontext auf. Die Lernplattform Moodle kommt laut den Kindern punktuell zum Einsatz, wenn es darum geht, die HomeschoolingInhalte zu organisieren:

B1: Da kann Frau (unv?) dann den Stundenplan einstellen.

Die Interviews zeigen demnach, dass seitens der Lehrkräfte offenbar nicht so sehr der Einsatz digitaler Medien im Fokus der ersten Homeschoolingphase stand, sondern das Zurverfügungstellen unterschiedlicher analoger Medien bzw. Materialien (A4: Ja mit Heften, mit Arbeitsblättern, mit, ja, allen möglichen Sachen halt) und Lernangebote, die haptische Eigenschaften und konkretes Anschauungsmaterial umfassen und an den Schüler*innen bisher bekannte Aufgabenformate anknüpfen:

B1: Also ich habe, unsere Lehrerin hat uns da so riesige Umschläge mit Din A4 Blättern gemacht und die durften wir dann machen, die hat sie uns in den Briefkasten gelegt, die konnte man dann rausholen und die Hausaufgaben dann machen, bei mir waren sie immer schon auf dem Schreibtisch, weil meine Mama und mein Papa sie immer reinbringen und dann habe ich halt am ersten Tag angefangen mit Mathe.

Dies setzt zum einen an grundlegende Bedingungen grundschulischen Lernens an und zeigt zugleich auf, mit welcher Plötzlichkeit das Homeschooling seitens der Lehrkräfte ohne weitere Vorbereitungen - sei es auf das digitale Lehren oder bloße organisatorische Aspekte wie die Hardwarebeschaffung etc. - organisiert werden musste.

I: Und wie habt ihr dann gelernt oder womit habt ihr gelernt?

A2: Also (.) ich saß an meinem Schreibtisch und ich hatte so eine Rechenkette, da haben wir auch erst bis 10 gerechnet, manchmal sogar bis 20 , aber ich hatte halt so eine 
Rechenkette, da waren 10 (.) solche Kugeln dran und damit habe ich am meisten gerechnet, weil es war am einfachsten.

I: Hm okay. Womit hast du gelernt?

B2: Ich habe halt im Kopf nachgedacht.

I: Hm. (..) Sonst irgendwas anderes? Was ihr sonst noch so an Unterlagen hattet oder an Materialien?

A2: Naja wir haben immer von der Frau B. manche Hilfssachen, zum Beispiel wie so Hilfsgeld oder so. (...) 2B: Ja und wir haben dann auch, ähm, Anleitung, weil da haben wir dann auch so Sachen bekommen, was wir denn zum Beispiel basteln können.

Die Aussagen der Kinder zeigen, dass die schulischen Lern- und Materialangebote individuell in die Gegebenheiten, die jeweils vor Ort in den Familien zur Verfügung stehen, eingebettet werden. Dabei werden auch handlungsorientierte Lernzugänge mit analogen Medien präferiert, die durch den Einsatz des „Hilfsgelds“ sowie Bastelangebote deutlich werden.

\section{Herausforderungen bäuslicher Lernumwelten}

Durch den Übertrag ähnlicher Arbeitsweisen aus dem regulären Schulalltag und mittels des Einsatzes analoger Medien ergeben sich im heimischen Umfeld aus Sicht der Kinder auch Erschwernisse, die so im schulischen Kontext nicht auftreten (und durch den Einsatz digitaler Tools gegebenenfalls entkräftet hätten werden können). Beispielsweise zeigt sich in den Interviews mit den Zweitklässler*innen, dass die veränderten schulischen Logiken im familialen Umfeld zunächst individueller Anpassungsstrategien seitens der Kinder bedürfen, da sich verschiedene Herausforderungen ergeben, die das eigene Lernen deutlich behindern (z.B. Geschwisterkinder, Arbeitsplatz einrichten). Als einen wesentlichen Unterschied zum schulischen Lernen wird u.a. das Lernen alleine zuhause benannt, das durchgängig seitens der Schulanfänger*innen als Nachteil wahrgenommen wird. Denn während die Gruppenprozesse und das Fehlen der Peers bzw. Mitschüler*innen besonders im Fokus der wahrgenommenen Nachteile der Homeschoolingphasen stehen, kommen potenzielle Vorteile des alleine Lernens - wie beispielsweise bessere Konzentrationsmöglichkeiten und weniger Ablenkung - im familialen Alltag nur bedingt und abhängig vom familiären Kontext zum Tragen. Insbesondere Aushandlungsprozesse zu unterschiedlichen Bedürfnissen und Aufgaben mit anderen Familienmitgliedern bzw. allein deren Anwesenheit scheinen aus Sicht der Kinder fordernd und erschweren die schulischen Tätigkeiten im heimischen Umfeld:

B1: Nämlich, dass ähm, mein Papa dann auch noch zuhause gearbeitet und meine Mama und da war dann halt ganz viele laute Stimmen, weil die hatten auch Gespräche und in der Schule war es halt viel leiser.

So ist das „peer-lose“ Lernen oftmals nur unter erschwerten Bedingungen möglich, da andere Familienmitglieder mit im Haushalt sind, deren unterschiedliche Bedürfnisse kollidieren und das individuelle Bearbeiten von Aufgaben zusätzlich erschweren:

A2: Ja ich habe alleine gelernt, aber mein Bruder war ständig bei mir, weil er wollte halt mit mir spielen und der musste ja auch zuhause bleiben. 
B2: Meine Schwester war genau so, die hat mich dann die ganze Zeit genervt, weil die erst zwei ist.

Jüngere Geschwisterkinder werden wiederholt als Herausforderung benannt, mit der während der Homeschoolingphasen umgegangen werden musste:

B2: Und sonst habe ich gut gelernt, außer wenn mal an manchen Tagen mich meine Schwester nerven.

Familienwelt und Lernumgebung müssen im Homeschooling neu ausgehandelt werden. Dass aber gleichzeitig kreative Strategien zur Aneignung des neuen häuslichen Lernkontexts von den Kindern genutzt werden, wird noch im Folgenden aufgezeigt.

\section{Lernen obne Peers und Lebrkraft}

Insgesamt wurde es von allen Kindern als herausfordernd erlebt, dass das Lernen alleine vonstattengehen musste und insbesondere im Frühsommer 2020 weder Mitschüler*innen noch die Lehrkraft unmittelbar während der Lernsituationen unterstützend zur Seite standen:

A2: Naja, es wurde halt nicht vorne an der Tafel gezeigt, sondern einfach wir mussten das allein im Kopf machen oder manchmal Mama und Papa fragen. Aber die mussten ja von zuhause auch arbeiten. (...)

B2: Konnte man nicht einfach die Lehrerin fragen.

Der fehlende direkte Austausch mit der Lehrkraft wird wiederholt als Manko angeführt, da bei direkten Rückfragen nicht auf die Kompetenz der Lehrkraft bzw. der Mitschüler*innen zurückgegriffen werden kann:

B1: Weil in der Schule, da sind ja auch ganz viele andere Kinder dabei und da sitzt man halt an einem festen Platz und bei sich zuhause ist halt nur ein Kind und man lässt sich ja auch mehr ablenken als in der Schule, find ich.

Auch wenn die Lehrkraft in dieser ersten Phase im regelmäßigen Kontakt mit den Schüler*innen ihrer Klasse stand, konnte die digitale Plattform zunächst nicht genutzt werden, um eben solche Interaktionsmöglichkeiten zwischen Schüler*innen, Peers und Lehrkraft zu schaffen. In den folgenden Homeschoolingphasen, so zeigt auch eine weitere Erhebung im Juni 2021, kam Moodle jedoch zunehmend als Rahmung für die täglichen Lerneinheiten zu Hause zum Einsatz.

Des Weiteren zeigt sich, dass der bloße Übertrag schulischer Materialien aus Sicht der Schüler*innen keinen Ersatz für schulisches Lernen darstellt, da das schulische Setting für die Schüler*innen primär durch die Anwesenheit der Lehrkraft und der Mitschüler*innen sowie das konkrete räumliche Setting (Tafel, Schulbänke etc.) geprägt sind:

B1: Ähm, dass man halt nicht mit seinen Freunden lernen konnte, dass man die Lehrerinnen nicht sofort fragen kann und oder sich melden kann und eine Antwort sagen kann, man kann ja in der Schule unsere Lehrerin, wenn wir Arbeitsblätter bearbeiten müssen, dann macht sie uns manchmal eine kleine [Beispielaufgabe oder hinterlegt 
Tippkarten], dann arbeiten wir die zusammen noch am Ende, dann können wir sie sozusagen überprüfen, das hat halt bei uns keiner gemacht, das war halt deswegen haben wir Hilfe gebraucht.

Zwar werden wiederholt die Eltern als Unterstützer*innen benannt, allerdings werden diese Hilfsangebote von einzelnen Kindern als nicht ausreichend beschrieben:

B2: Aber ein Ding habe ich nicht verstanden so und das fand ich dann ganz doof, weil man sich da nicht mehr melden kann in der Schule und Fragen stellen, weil Mama und Papa wissen ja nicht genau, wie das sein soll, weil meistens fragen die mich, wie das geht.

Die Eltern sind hier in einer besonderen Rolle: Einerseits teilweise in eigene Arbeitsprozesse des Homeoffice eingespannt, die es mit dem familiären Rahmen auszuhandeln gilt, andererseits als Ersatz-Lehrkraft vor Ort gefragt, um bei den Aufgaben zu unterstützen und Rückmeldung zu geben. Die Aussagen der Kinder zeigen zudem auf, dass die Eltern auch die Rolle einer fragenden Lehrkraft übernehmen (,[...] weil meistens fragen die mich, wie das geht“"). Die Vorstellungen von schulischem Lernen der Eltern, die wohl - so kann man vermuten - größtenteils auf die Erfahrungen aus der eigenen Schulzeit zurückgreifen, stehen dabei nicht immer im Einklang mit dem Verständnis von schulischem Lernen wie es die Kinder bisher in ihrer Grundschule erlebt haben. So werden beispielsweise von der Lehrkraft empfohlene Anwendungen, Filme und Apps (Anton-App, Trickfilme), die eigentlich in den unterrichtlichen Ablauf eingebettet werden sollten, von den Eltern eher als Belohnung hinter die Bearbeitung schulischer Aufgaben gesetzt und damit insbesondere neue Medien seitens der Eltern weniger als Lehr-Lern-Format, sondern vielmehr als Unterhaltungselement mit Belohnungseffekt bestimmt.

\section{Kein Unterricht, sondern nur noch „Hausis“}

Ein Hinweis dafür, dass die Aufgabenbearbeitungen im heimischen Umfeld als Einzelperson ohne Gruppenkontext von den Kindern nicht als Schule und Unterricht im engeren Sinne wahrgenommen werden, ist die fast durchgängige Bezeichnung der unterrichtlichen Aufgabenstellungen seitens der Kinder als „Hausis“. Während Hausaufgaben im alltäglichen Schulverständnis vor der Covid-19-Pandemie als Bezeichnung für diejenigen Aufgabenformate üblich sind, die der individuellen Vertiefung zuhause dienen, verschmelzen für die Kinder in den Homeschoolingphasen die Unterrichtseinheiten mit den Hausaufgaben zu einem:

B4: Ich fand das voll blöd.

I: Kannst du erklären, warum? Ich nehme dich gleich dran.

B4: Weil (.) ich gar nicht mehr meine Freunde gesehen habe und die Frau B. gar nicht und so.

I: Mhm (bestätigend).

B4: (Holt Luft) alle einfach gar nicht.

I: Mhm (bestätigend). 
A4: Also ich fand es auch so. Es waren halt mehr Hausis, weil wir dann auch die Schulaufgaben UND gleichzeitig die Hausis, ähm, zu Hause machen mussten. (...)

I: Was war denn im Homeschooling schwerer als in der Schule?

B4: Dass wir halt so viele Hausi hatten. (...)

B4: Aber auch der Stundenplan, der war viel, viel anders.

I: Mhm (bestätigend), kannst du das erklären? Was war da anders?

B4: DASS halt (.), dass (schluckt), dass wir, ähm, (.), dass halt auf dem (.), ähm, Hausiplan halt stand, ähm, (holt Luft) wir sollten jetzt das, das, das und das machen. (Holt Luft) und das halt im Indianerheft vor allem und im Zahlenfuchs, dass wir das auch machen sollten. (...)

Die Verschmelzung von familiärem und schulischem Lernen bildet sich hierbei ab; da der Unterricht im Homeschooling in dieser ersten Phase - ähnlich wie Hausarbeiten - vorrangig durch Arbeitsblätter und andere analoge Materialien organisiert wurde, war für die Kinder keine Grenze erkennbar.

\section{Die Dinge als Mittler - „Spacing“ als Aneignungsstrategie}

Neben der Herausforderung Aufgaben zuhause alleine zu bearbeiten, zeigte sich in den Interviews, dass auch das Fehlen der schulischen Räumlichkeiten als Herausforderung wahrgenommen wurde. Dies bewältigten die Kinder u.a. dadurch, dass sie ihre heimischen Räumlichkeiten sowohl faktisch als auch imaginär den Klassenzimmern anzugleichen versuchten. Dies erfolgte zum einen durch die Imagination einer Tafel:

A2: Also ich habe mir halt so eine ganz große Tafel vor mir vorgestellt und (..) Ich habe mir vorgestellt, dass da meine Aufgabe ist und ich jetzt drangenommen wurde und das Ergebnis sagen musste.

Aber auch durch das Spielen von Schule mit Geschwisterkindern,

A2: und dann in der ganzen Corona Zeit haben mein Bruder und ich auch gern Schule gespielt.

durch fiktive Klassenkamerad*innen in Form von Spielfiguren

A4: Also ich habe dann einfach so gelernt und hatte Gesellschaft von meinen (Legorittern?) und manchmal auch von meinen Kuscheltieren (lacht).

und zeitweise Unterstützung der Eltern

A4: (...). Ja, meine Mama hat immer Lehrerin gespielt und hat eine Tafel aufgebaut.

Innerhalb der interdependenten Triade kindlichen Lernens „Ich und Selbst“, „Ich und Andere" wird hier nun auch die dritte Dimension „Ich und die Dinge“ - nämlich der Raum (als „dritter Erzieher") - bedeutsam (vgl. Dietrich 2011; Nohl 2011): Die Kinder nutzen hierbei die „Dinge“ als Ko-Akteure, um eine soziale Ordnung, nämlich die vertraute schulische Ordnung (Lehrkraft - Schüler*in), herzustellen (vgl. Grunau/Kekeritz 2015). Gleichzeitig werden in einer Positionierung zwischen Fantasie und Gegenständlichkeit häusliche Dinge aus 
dem eigenen Kinderzimmer, wie Spielfiguren oder Kuscheltiere, „entfunktionalisiert“ und gewissermaßen „pädagogisiert“ (Neumann 2012, S. 169), um schulische Situationen herzustellen.

Im Raum Zuhause ist durch das Homeschooling ein weiterer Raum entstanden (vgl. Löw 2001) und dieser Raum als eine „relationale (An-)Ordnung von Lebewesen und sozialen Gütern an Orten” wird durch „das Errichten, Bauen oder Positionieren“ (Löw 2001, S. 271) von sozialen Gütern wie materiellen Güter durch Individuen (vgl. Löw 2001, S. 153) angeeignet: Erst diese vom Kind hergestellten räumlichen Arrangements im Sinne eines Spacings (vgl. Deinet 2004) „institutionalisieren“ den häuslichen Kontext und lassen damit für die Kinder schulische Lernsituationen entstehen.

Die Dinge werden damit in phänomenologischer Perspektive dazu genutzt, um die ihnen bekannte Ordnung Lehrkraft - Kind zu reproduzieren (vgl. Corsaro 2012), schulische (Lern-)Situationen herzustellen und gleichzeitig einen neuen Möglichkeitsraum zu schaffen und kreativ zu nutzen.

In dieser räumlich-materiellen Aneignung der Kinder zeigt sich eine kreative und selbstaktive Strategie der Kinder mit der herausfordernden Situation des Homeschoolings umzugehen. Die Interaktionen mit Lehrkraft und Peers, die den Kindern im Homeschooling misslich fehlen, werden auf anderer Ebene hergestellt und eben durch diese Interaktionen die Bedeutung einer institutionellen Rahmung, auch auf räumlich-materieller Ebene, lesbar.

\section{Diskussion und Ausblick}

Im Kontext der ersten Homeschoolingphase verweisen die Ergebnisse insgesamt sowohl auf individuell als auch kollektiv wahrgenommene Herausforderungen, gleichzeitig aber auch auf kreative Praktiken im Sinne von Bewältigungsstrategien der Kinder im Umgang mit diesen Aufgabenstellungen. Insbesondere die rekonstruierten Herausforderungen durch den Wegfall von unterrichtsbegleitenden Interaktionen mit der Lehrkraft und den Peers könnten zukünftig durch die Einbindung digitaler Angebote, die einen unmittelbaren Austausch zwischen Mitschüler*innen und Lehrpersonen überhaupt erst ermöglichen, umgesetzt werden. Dies bedarf jedoch didaktischer Rahmungen und Konzeptionen, die Lernangebote für Kinder im Grundschulalter synergetisch mit digitalen und analogen Medien im Sinne einer partizipativ ausgerichteten Medienbildung (vgl. Hüpping/Kamin 2020) verbindet. So bieten z.B. Ansätze eines forschenden Lernens eine Möglichkeit, die Erfahrungen und das Wissen der Kinder zu bündeln und Beobachtungsaufträge (z.B. das Entdecken geometrischer Grundformen in der Lebenswelt der Kinder im Kontext eines fachübergreifenden Kunst- und Mathematikangebots) mit digitalen Tools zu verknüpfen. Zudem könnten die für die Grundschule postulierten projektorientierte Lernzugänge mit Formen einer aktiven Medienarbeit (vgl. Kamin/Meister/Sonnenschein 2018, S. 182) verknüpft werden, um den integrativen Bestandteil digitaler Medien auch auf die Prozess- und Produktorientierung auszuweiten.

Die Ergebnisse verdeutlichen weiter, wie die Kinder Praktiken schulischen Lernens im familiären Umfeld aufgreifen, übersetzen und anwenden. Erinnert sei hier an die gestalteten Lernsettings durch die Kinder (z.B. das Aufstellen einer Tafel), an die Art und Weise der zu bearbeitenden handlungsorientierten Aufgaben sowie den Stellenwert analoger und digitaler 
Medien. Exemplarisch lässt sich durch den institutionellen Zugriff eine Verschiebung schulischer Praktiken in Richtung der Familien aufzeigen, die eng mit individuellen Übersetzungsleistungen der Kinder und ihrer Eltern verwoben sind und im wahrsten Sinne des Wortes Raum einnehmen und den Familienalltag stark beeinflussen. Dies zeigt sich insbesondere in den fehlenden Interaktionen mit den Peers und der Klassenlehrerin, die durch Geschwisterkinder und Eltern nur bedingt ersetzt werden konnten.

Spezifisch die im ersten Lockdown angeführte Abwesenheit interaktiver Lernmöglichkeiten, das Fehlen einer gemeinsamen Teilhabe an Unterrichtseinheiten und damit ausbleibende Kommunikation untereinander waren aus Sicht der Kinder im heimischen Lernsetting besonders nachteilig und belastend. Wenngleich es langfristig grundlegender Veränderungen in Bezug auf Lehr- und Lernprozesse in der digitalen Welt bedarf, könnten medienpädagogisch aufbereitete Angebote und der Einsatz digitaler Tools unter Mitschüler*innen bzw. mit der Lehrkraft zumindest den unmittelbaren Kommunikationscharakter von Schule und Unterricht auch zum gegenwärtigen Zeitpunkt bereits sinnvoll ergänzen helfen. Allerdings zeigen die vorliegenden Interviews aus den ersten Homeschoolingphasen das, worauf Huber et al. (vgl. 2020) verweisen, nämlich, dass der Anteil des analogen Arbeitens insbesondere in niedrigeren Schulstufen sehr hoch ist. Dies wird u.a. damit begründet, dass die „Entwicklung sozioemotionaler Kompetenzen und des Austauschs untereinander [...] eingeschränkter möglich“ (Huber et al. 2020, S. 109) sei. Vor dem Hintergrund der vorliegenden Erkenntnisse könnte jedoch auch anders argumentiert werden, nämlich dass gerade jüngere Grundschulkinder den sozialen Kontext und das interaktive Lernangebot benötigen, wenn es um Homeschooling geht, da das analoge Lernen an konkreten Materialien Unterricht als soziales Setting nur unzureichend abbilden kann und zu den rekonstruierten Herausforderungen aus Sicht der Kinder führt. Folgt man dieser Logik, so wird insbesondere das Potenzial digitaler Medien virulent, welches dem interaktiven Austausch und den sozialen Kontakten untereinander dient - sowohl mit den Mitschüler*innen als auch mit den Lehrkräften. Dies knüpft zudem an die genannten Überlegungen von Irion und Scheiter (vgl. 2018) an, die darauf hinweisen, dass digitale Medien neben ihren Potenzialen im Rahmen von Präsentation und Individualisierung u.a. im Hinblick auf die Kooperationsmöglichkeiten der Schüler*innen untereinander pädagogisch sinnvoll sein können.

Der integrative Bestandteil digitaler Medien bietet somit die Chance, Synergien zwischen analogen und digitalen Lernmodalitäten herzustellen. Hierfür kann eine partizipativ ausgerichtete Medienbildung einen Beitrag leisten, um somit die Vermittlung eines reflexiven und verantwortungsbewussten Umgangs mit und über Medien ausreichend zu berücksichtigen (vgl. Petko 2014, S. 157).

\section{Literatur}

Anders, Florentine (2021): Online lernen. Wie gelingt Distanzunterricht? Das Deutsche Schulportal. URL: deutsches-schulportal.de/unterricht/angebote-fuer-das-lernen-zu-hause (16.08.2021).

Bollig, Sabine/Kelle, Helga (2014): Kinder als Akteure oder als Partizipanden von Praktiken? Zu den Herausforderungen für eine akteurszentrierte Kindheitssoziologie durch Praxistheorien. In: ZSE Zeitschrift für Soziologie der Erziehung und Sozialisation 34 (3), S. 263-279. 
de Boer, Heike/Deckert-Peaceman, Heike (2009a): Kinder in der Schule. Zwischen Gleichaltrigenkultur und schulischer Ordnung. Wiesbaden: VS Verlag für Sozialwissenschaften.

de Boer, Heike/Deckert-Peaceman, Heike (2009b): Kinder und Schule - Rekonstruktionen der kindlichen Perspektive und ihre Bedeutung für die schulische Ordnung. Wiesbaden: VS Verlag für Sozialwissenschaften, S. 21-34.

Corsaro, William A. (2012): Interpretive Reproduction in Children's Play. In: American Journal of Play, 4 (4), pp. 488- 504.

Deinet, Ulrich (2004): „Spacing“, Verknüpfung, Bewegung, Aneignung von Räumen - als Bildungskonzept sozialräumlicher Jugendarbeit. In: Deinet, Ulrich/Reutlinger, Christian (Hrsg.): „Aneignung“ als Bildungskonzept der Sozialpädagogik. Beiträge zur Pädagogik des Kindes- und Jugendalters in Zeiten entgrenzter Lernorte. Opladen: Leske + Budrich, S. 175-190.

Dietrich, Cornelie (2011): Bildungstheoretische Notizen zur Beobachtung frühkindlicher Bildungsprozesse. In: Cloos, Peter/Schulz, Marc (Hrsg.): Kindliches Tun beobachten und dokumentieren. Perspektiven auf die Bildungsbegleitung in Kindertageseinrichtungen. Weinheim: Beltz Juventa, S. $100-114$.

Eßer, Florian (2014): Agency Revisited. Relationale Perspektiven auf Kindheit und die Handlungsfähigkeit von Kindern. In: ZSE Zeitschrift für Soziologie der Erziehung und Sozialisation 34 (3), S. 233-246.

Eickelmann, Birgit (2019): ICILS 2018 \#Deutschland: Computer- und informationsbezogene Kompetenzen von Schülerinnen und Schülern im zweiten internationalen Vergleich und Kompetenzen im Bereich Computational Thinking. Münster/New York: Waxmann.

Fickermann, Detlef/Edelstein, Benjamin (2020a): „Langsam vermisse ich die Schule ...“. Schule während und nach der Corona-Pandemie. In: Die Deutsche Schule, 16. Beiheft. Münster: Waxmann.

Fickermann, Detlef/Edelstein, Benjamin (2020b): Editorial. In: Fickermann, Detlef/Edelstein, Benjamin (Hrsg.): „Langsam vermisse ich die Schule ...“. Schule während und nach der Corona-Pandemie. Die Deutsche Schule, 16. Beiheft. Münster: Waxmann, S. 9-33.

Glaser, Barney G./Strauss, Anselm L. (1998): Grounded Theory. Strategien qualitativer Forschung. Bern: Verlag Hans Huber.

Grunau, Thomas/Kekeritz, Mirja (2015): „Verdammtes Ding, dich könn’ wir dann erforschen!“ Perspektiven auf kindliche Auseinandersetzungen mit den Dingen in institutionellen Kontexten. In: Zeitschrift für interpretative Schul- und Unterrichtsforschung (ZISU) 4, S. 31-47.

Hartnack, Florian (2019): Qualitative Forschung mit Kindern: Herausforderungen, Methoden und Konzepte. Wiesbaden: Springer VS.

Heinzel, Friederike (2019): Zur Doppelfunktion der Grundschule, dem Kind und der Gesellschaft verpflichtet zu sein - die generationenvermittelnde Grundschule als Konzept. In: Zeitschrift für Grundschulforschung, 12 (2), S. 275-287. URL: 10.1007/s42278-019-00050-x (16.08.2021).

Huber, Stephan G./Günther, Paula Sophie/Schneider, Nadine/Helm, Christoph/Schwander, Marius/Schneider, Julia A./Pruitt, Jane (2020): COVID-19 und aktuelle Herausforderungen in Schule und Bildung. Erste Befunde des Schul-Barometers in Deutschland, Österreich und der Schweiz. Münster/New York: Waxmann Verlag.

Hüpping, Birgit/Kamin, Anna-Maria (2019): Partizipation durch Medienbildung - Medienbildung durch Partizipation: partizipative Medienbildung in der Grundschule. In: Zeitschrift für Theorie 
und Praxis der Medienbildung, 39, S. 121-141.

Idel, Till-Sebastian/Rabenstein, Kerstin/Reh, Sabine (2013): Transformation der Schule - praxistheoretisch gesehen. Rekonstruktionen am Beispiel von Familiarisierungspraktiken. In: Rürup, Matthias/Bormann, Inka (Hrsg.): Innovationen im Bildungssystem. Analytische Zugänge und empirische Befunde, Wiesbaden: VS Verlag, S. 249-268.

Irion, Thomas/Scheiter, Katharina (2018): Didaktische Potenziale digitaler Medien. Der Einsatz digitaler Technologien aus grundschul- und mediendidaktischer Sicht. In: Grundschule aktuell, 142, S. 8-11.

Kamin, Anna-Maria/ Meister, Dorothee M. /Sonnenschein, Nele (2018): Medienpädagogik in pädagogischen Grenzfeldern - konzeptionelle Überlegungen am Beispiel des Maßregel-vollzugs. In: Knaus, Thomas/Engel, Olga (Hrsg.): Spannungen und Potentiale - Digitaler Wandel in Bildungseinrichtungen (6. Band der fraMediale-Reihe). München: kopaed, S. 175-193.

Kekeritz, Mirja (2017): Didaktische Interaktionen im Übergang zur Grundschule. Zum Wechselspiel von Kontinuität und Neubeginn. Wiesbaden: Springer VS.

KMK - Kultusministerkonferenz (2017): Bildung in der digitalen Welt. Strategie der Kultusministerkonferenz. Bonn. URL: kmk.org/fileadmin/Dateien/pdf/PresseUndAktuelles/2018/Digitalstrategie_2017_mit_Weiterbildung.pdf(09.06.2021).

Knörzer, Martina/Förster, Lars/Hartinger, Andreas/Franz, Ute (2019): Forschendes Lernen im Sachunterricht. Probleme und Perspektiven des Sachunterrichts (Band 29). Bad Heilbrunn: Klinkhardt.

Konecki, Krzysztof Tomasz (2011): Visual Grounded Theory: A Methodological Outline and Examples from Empirical Work. In: Revija za Sociologiju, 41 (2), pp. 131-160.

Löw, Martina (2001): Raumsoziologie. Frankfurt am Main: Suhrkamp.

MPFS - Medienpädagogischer Forschungsverbund Südwest (2018): KIM-Studie 2018. Kindheit, Internet, Medien. Basisuntersuchung zum Medienumgang 6- bis 13-Jähriger. URL: mpfs.de/filead $\mathrm{min} /$ files/Studien/KIM/2018/KIM-Studie_2018_web.pdf(09.06.2021).

MPFS - Medienpädagogischer Forschungsverbund Südwest (2020): KIM-Studie 2020. Kindheit, Internet, Medien. Basisuntersuchung zum Medienumgang 6- bis 13-Jähriger. URL: mpfs.de/filead min/files/Studien/KIM/2020/KIM-Studie2020_WEB_final.pdf(09.06.2021).

Neumann, Sascha (2012): Pädagogisierung und Verdinglichung. Beobachtungen zur Materialität der Frühpädagogik. In: Priem, Karin/König, Gudrun M./Casale, Rita (Hrsg.): Die Materialität der Erziehung. Kulturelle und soziale Aspekte pädagogischer Objekte. Weinheim/Basel: Beltz Verlag, S. $168-184$.

Nohl, Arnd-Michael (2011): Pädagogik der Dinge. Bad Heilbrunn. Klinkhardt.

Petko, Dominik (2014): Einführung in die Mediendidaktik: Lehren und Lernen mit digitalen Medien (Reihe „Bildungswissen Lehramt“ 25), Weinheim: Beltz.

Ravens-Sieberer, Ulrike/Kaman, Anne/Otto, Christiane/Adedeji, Adekunle/Devine, Janine/Erhart, Michael/Napp, Ann-Kathrin/Becker, Marcia/Blanck-Stellmacher, Ulrike/Löffler, Constanze/ Schlack, Robert/Hurrelmann, Klaus (2021): Seelische Gesundheit und psychische Belastungen von Kindern und Jugendlichen in der ersten Welle der COVID-19-Pandemie - Ergebnisse der COPSY-Studie. In: Bundesgesundheitsblatt - Gesundheitsforschung - Gesundheitsschutz. URL: doi.org/10.1007/s00103-021-03291-3 (09.06.2021). 
Schaumburg, Heike (2019): Chancen und Risiken digitaler Medien in der Schule. Medienpädagogische und -didaktische Perspektiven. In: Bertelsmann Stiftung (Hrsg.): Individuell fördern mit digitalen Medien. Chancen, Risiken, Erfolgsfaktoren. 3. Auflage, Gütersloh: Bertelsmann Stiftung, S. 19-94.

van Gennep, Arnold (2005): Übergangsriten (= Les rites de passage). Aus dem Französischen von Klaus Schomburg und Sylvia M. Schomburg-Scherff. Frankfurt am Main/New York: Campus.

\section{Informationen zu den Autorinnen}

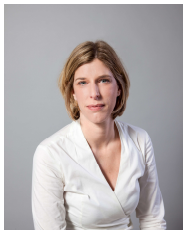

Dr. phil. Birgit Hüpping, Professorin für Erziehungswissenschaft mit Schwerpunkt Pädagogik und Didaktik des Primarbereichs, Pädagogische Hochschule Ludwigsburg. Arbeitsschwerpunkte: Partizipation, Heterogenität in Bezug auf Migration und Inklusion, qualitative Forschungszugänge.

birgit.huepping@ph-ludwigsburg.de

Dr. phil. Mirja Kekeritz arbeitet am Institut für Erziehungswissenschaft der Universität Osnabrück. Schwerpunkte in der Forschung und Lehre: ästhetische Bildungsformen der Kindheit, Methoden der Kindheitsforschung, Interaktionen zwischen Pädagog*innen und Kindern, Individualisierung von Grundschulunterricht sowie Übergang KiTaGrundschule.

mirja.kekeritz@uni-osnabrueck.de

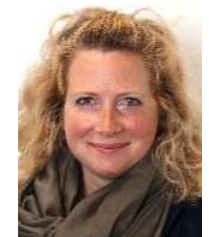

Dr. phil. Melanie Kubandt, Juniorprofessorin für Gender und Bildung, Universität Vechta. Arbeitsschwerpunkte: Professionalität und Professionalisierung in der Pädagogik der (frühen) Kindheit, Gender und Diversität im Kontext von (frühen) Bildungsinstitutionen, Sozialdidaktik, qualitativ-rekonstruktive Forschungszugänge.

melanie.kubandt@uni-vechta.de

\section{Zitationshinweis:}

Hüpping, Birgit/Kekeritz, Mirja/Kubandt, Melanie (2021): Unterricht zwischen Schule und Zuhause: Perspektiven von Schulanfänger*innen auf Lernprozesse im Homeschooling. In: Online-Magazin Ludwigsburger Beiträge zur Medienpädagogik, Ausgabe 21/2021. URL: medienpaed-ludwigsburg.de/ 